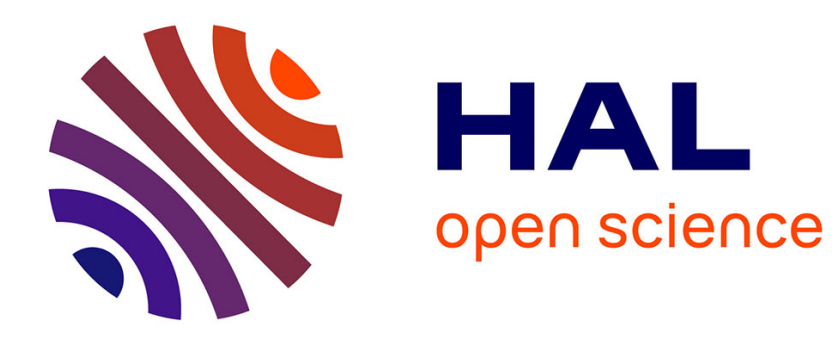

\title{
Sur le rôle des milieux diélectriques en électrostatique M. Gouy
}

\section{- To cite this version:}

M. Gouy. Sur le rôle des milieux diélectriques en électrostatique. J. Phys. Theor. Appl., 1896, 5 (1), pp.154-159. 10.1051/jphystap:018960050015401 . jpa-00239860

\section{HAL Id: jpa-00239860 https://hal.science/jpa-00239860}

Submitted on 1 Jan 1896

HAL is a multi-disciplinary open access archive for the deposit and dissemination of scientific research documents, whether they are published or not. The documents may come from teaching and research institutions in France or abroad, or from public or private research centers.
L'archive ouverte pluridisciplinaire HAL, est destinée au dépôt et à la diffusion de documents scientifiques de niveau recherche, publiés ou non, émanant des établissements d'enseignement et de recherche français ou étrangers, des laboratoires publics ou privés. 
SUR LE ROLE DES MILIEUX DIÉLEGTRIQUES EN ÉLEGTROSTATIQUE (1);

Pår M. GOUY.

1. Dans la théorie classique de l'électrostatique, telle qu'elle a été conçue à l'origine, la force électrostatique $f$, qui s'exerce entre deux particules électriques données, ne dépend que de leur distance. Depuis lors, l'expérience et de simples considérations d'énergie ont appris que les forces apparentes qui s'exercent entre des conducteurs électrisés, de charges données, sont en raison inverse du pouvoir inducteur $\mathrm{K}$ du diélectrique liquide ou gazeux qui remplit le champ. Pour en rendre compte $\left({ }^{2}\right)$, on admet souvent que $f$ est en raison inverse de $\mathrm{K}$, en sorte que le coefficient $\varepsilon$, qui entre dans la formule élémentaire :

$$
f=\varepsilon \frac{m m^{\prime}}{r^{2}}
$$

(1) Ce travail est le développement d'une note parue aux Comples Rendus de l'Académie des Sciences, le $1^{\text {er }}$ juillet 1895.

${ }^{(2)}$ La considération de la force du champ, ou force électrique, dans le diélectrique donne un semblant d'explication; mais cette quantité, au sein d'un diélectrique polarisable, est définie d'une manière conventionnelle, et ne peut servir, comme dans un milieu non polarisable, à calculer l'action électrostatique des masses agissantes, parmi lesquelles il faut comprendre les éléments du diélectrique lui-même. 
ne serait pas une constante absolue, mais dépendrait du milieu où se propage la force électrostatique.

Cette manière de voir soulève plusieurs difficultés. Elle conduit d'abord à des résultats contradictoires, si l'on considère autre chose que le cas simple des attractions ou répulsions mutuelles de conducteurs indéformables, placés dans un même milieu.

On doit remarquer aussi que cet énoncé ne peut résulter des expériences électrostatiques qu'après une discussion préalable. Si, en effet, on adopte l'hypothèse classique de l'électricité et des forces électrostatiques(comme une fiction mathématique permettant de coordonner l'électrostatique), c'est pour en développer les conséquences, en raisonnant comme si elle exprimait une réalité. Dès lors, l'expérience nous fournit, non pas une définition de $f$, mais un moyen de mesurer cette quantité. Cette mesure sera correcte si $f$ est la seule force qui agisse sur les conducteurs électrisés, où nous constatons des forces apparentes. S`il y a d'autres forces mises en jeu avec $f$, il faudra les déduire du résultat brut de la mesure, pour avoir la valeur de $f$.

Je vais essayer de montrer que l'existence de ces forces perturbatrices résulte, en effet, de la théorie ordinaire de la polarisation diélectrique et que, si on en tient compfe, on trouve que l'expérience est d'accord avec l'hypothèse que $f$ est indépendant du milieu ambiant, comme l'admettaient les fondateurs de l'électrostatique.

2. Il est utile de rappeler d'abord que, en général, le milieu fluide où se trouve placé un corps peut donner des forces apparentes, en produisant une pression hydrostatique différente aux divers points de sa surface. Cela aura lieu lorsque les éléments du milieu fluide seront eux-mêmes soumis à des forces tendant à les déplacer.

Considérons, par exemple, l'attraction newtonienne de deux sphères solides, de volumes $V_{1}$ et $V_{2}$ et de densités $\delta_{1}$ et $\delta_{2}$, au sein d'une très grande masse d'eau, de figure invariable et de densité $\delta_{0}$. La sphère 1 étant en équilibre, il faudra, si on éloigne la sphère 2 , appliquer à la première, pour la maintenir en équilibre, une force qui est l'attraction apparente $a$. La sphère 2 étant remplacée, lorsqu'on l'éloigne, par un égal volume d'eau, il semble qu'on doit avoir :

$$
a=\gamma \frac{\mathrm{V}_{1} \delta_{1}\left(\mathrm{~V}_{2} \delta_{2}-\mathrm{V}_{2} \delta_{0}\right)}{r^{2}},
$$

$\gamma$ désignant la constante newtonienne. Ce résultat est pourtant 
erroné, et $a$ doit évidemment s’annuler pour $\delta_{1}=\delta_{0}$. En réalité, cette formule donne bien la différence des attractions exercées, sur la sphère 1, par la sphère 2 et par l'eau qui le remplace; mais, en outre, la sphère 2 agit par attraction sur le milieu ambiant, et produit une pression hydrostatique qui s'exerce inégalement sur la surface de la sphère 1 et intervient ainsi dans la force apparente $a$.

Si $a$ était connu expérimentalement, et qu'on oubliât de tenir compte de cette pression hydrostatique, on serait obligé de conclure, comme on le fait en électrostatique, que la constante $\gamma$ dépend du milieu ambiant.

3. Considérons des conducteurs électrisés $1,2,3 \ldots$, placés dans le vide.

Le conducteur 1 peut être regardé comme soumis à des forces appliquées à chaque élément $d s$ de sa surface, dirigées suivant la normale extérieure, et égales à $2 \pi \sigma^{2} d s$, en appelant $\sigma$ la densité électrique.

Supposons maintenant que, les conducteurs restant de même, ainsi que leurs charges, le champ soit rempli d'un diélectrique fluide, de pouvoir inducteur K. Une particule électrique quelconque du conducteur 1 est sollicitée par des forces électrostatiques qui émanent des autres particules électriques existant sur les conducteurs et des éléments du diélectrique polarisé.

Ces dernières forces ont une résultante nulle. En effet, le diélectrique entier peut être divisé en couches comprises entre des surfaces équipotentielles infiniment voisines, et chacune de ces couches forme un feuillet diélectrique fermé, qui ne produit de force électrique que dans son épaisseur ( ${ }^{1}$ ).

Il en résulte que la distribution sur les conducteurs est la même que précédemment. De plus, comme, par hypothèse, $f$ ne dépend pas du milieu ambiant, les forces électrostatiques qui agissent sur la charge du conducteur 1 sont les mêmes que tout à l'heure, dans le vide; il y a donc, appliquée à l'élément $d s$, la même force $2 \pi \sigma^{2} d s$.

(1) Pour abréger, je raisonne ici comme si la charge du conducteur était hors du diélectrique, mais il n'est pas inutile d'examiner l'autre hypothèse.

Supposons que la charge soit localisée dans la couche du diélectrique qui est au contact du conducteur. Si cette charge est soumise, de la part de cette couche, à des forces électrostatiques, ces forces sont équilibrées par celles qui y retiennent la charge, et il n'y a pas à en tenir compte. En effet, pour un déplacement virtuel de la surface du conducteur, le travail de ces forces est nul, puisque la couche extrême du diélectrique se déplace avec elle. 
Reste à expliquer pourquoi la force apparente qui agit sur ce conducteur est $\mathrm{K}$ fois plus petite que dans le vide.

4. On sait que, dans l'air ou dans le vide, un petit corps diélectrique est attiré suivant la direction où l'intensité du champ s'accroît le plus vite. Cette attraction, d'après la théorie usuelle, est due aux forces électrostatiques agissant sur chaque élément du diélectrique polarisé, et elle existe de même pour un diélectrique qui remplit tout le champ, tel que nous l'envisageons. Le diélectrique est soumis à des forces pondéromotrices de deux sortes, qui résultent des forces électrostatiques qu'exercent, sur chaque élément polarisé : $1^{\circ}$ les charges des conducteurs; $2^{\circ}$ les autres éléments du diélectrique.

Occupons-nous d'abord des premières de ces forces pondéromotrices, considérées comme agissant seules.

Soit, en chaque point, F la force électrique produite par les charges seules des conducteurs. La polarisation I est de même sens que F, et l'on a :

$$
\mathrm{I}=\frac{1}{4 \pi} \frac{\mathrm{K}-1}{\mathrm{~K}} \mathrm{~F} \text {. }
$$

La force pondéromotrice produite par les charges des conducteurs est appliquée au centre de gravité de chaque élément $d x d y d z$, et a pour composantes:

$$
\begin{aligned}
& \mathrm{I} d x d y d z \frac{d \mathbf{F}}{d x}=\frac{1}{8 \pi} \frac{\mathrm{K}-1}{\mathrm{~K}} \frac{d \mathbf{F}^{2}}{d x} d x d y d z ; \\
& \mathrm{I} d x d y d z \frac{d \mathbf{F}}{d y}=\frac{1}{8 \pi} \frac{\mathrm{K}-1}{\mathrm{~K}} \frac{d \mathbf{F}^{2}}{d y} d x d y d z ; \\
& \mathbf{I} d x d y d z \frac{d \mathbf{F}}{d z}=\frac{1}{8 \pi} \frac{\mathbf{K}-1}{\mathbf{K}} \frac{d \mathbf{F}^{2}}{d z} d x d y d z .
\end{aligned}
$$

L'équilibre de cet élément exige qu'on ait, en chaque point du fluide, une pression hydrostatique $p$ donnée par l'équation :

$$
p=\frac{1}{8 \pi} \frac{\mathrm{K}-1}{\mathrm{~K}} \mathrm{~F}^{2}
$$

en supposant nulle la pression aux points où le champ est insensible, et faisant abstraction de la pesanteur.

5. Les forces pondéromotrices de seconde espèce seront négli- 
geables si $\mathrm{K}$ - I est une quantité très petite, car leur expression contient en facteur $\mathrm{I}^{2}$ ou $(\mathrm{K}-\mathbf{1})^{2}$; ce sera le cas des gaz (1).

Ces forces s'annulent encore, quelle que soit la constante $\mathrm{K}$, dans le calcul de la pression exercée sur un conducteur par un diélectrique liquide, qu'on peut supposer incompressible, vu la petitesse des pressions mises en jeu.

Pour le montrer, nous avons à appliquer les formules établies par M. A. Liénard $(\stackrel{2}{*}$ pour le calcul de la pression dans les aimants et les diélectriques. Dans le cas de notre diélectrique incompressible, on a l'énoncé suivant :

La pression $\mathrm{P}$ au sein du fluide est normale à l'élément sur lequel elle s'applique, et donnée par l'équation:

$$
\mathrm{P}=2 \pi \mathrm{I}^{2}\left\{\frac{1}{\mathrm{~K}-1}+\cos ^{2}(\mathrm{I}, \mathrm{N})\right\}
$$

en désignant par ( $\mathrm{I}, \mathrm{N}$ ) l'angle que fait la polarisation I avec la normale à l'élément.

A la surface d'un de nos conducteurs plongé dans le diélectrique, l'angle (I, N) étant égal à zéro, cette équation s'écrit:

$$
\mathrm{P}=2 \pi \mathrm{I}^{2} \frac{\mathrm{K}}{\mathrm{K}-1}=\frac{1}{8 \pi} \frac{\mathrm{K}-1}{\mathrm{~K}} \mathrm{~F}^{2}=p
$$

Ainsi il n'y a à tenir compte, pour la pression exercée sur un de nos conducteurs, que du terme $p$ qui résulte de l'action exercée sur le diélectrique par les charges des conducteurs, ce qui nous montre que le diélectrique est en équilibre de lui-même sous les actions mutuelles de ses éléments (3).

6. Revenons maintenant au conducteur 1. L'élément $d s$ subit, du fait de la pression hydrostatique $p$. une force dirigée suivant la nor-

(1) Je me propose de montrer, dans un autre travail, que la pression $p$ est la cause vraisemblable du phénomène qui a reçu le nom de contraction électrique des gaz, et qui consiste en une compression analogue à celle que produit la pesanteur. Il y a lieu aussi de tenir compte de $p$ dans la question de la tension des vapeurs saturées dans un champ électrique.

$\left.1^{2}\right)$ La Lumière électrique, mars et avril 1894. Ces formules sont déduites de la théorie classique de la polarisation magnétique ou diélectrique.

(3) Céci n'est vrai, bien entendu, que lorsque le diélectrique est limité, comme ici, par des surfaces équipotentielles. 
HOULLEVIGUE. - GHALEUR DE VAPORISATION. 199 male intérieure, et égale à $\frac{1}{8 \pi} \frac{\mathrm{K}-1}{\mathrm{~K}} \mathrm{~F}^{2}$ cls. D autre part, les forces électrostatiques directes produisent sur $d s$ une force opposée à la précédente el égale à $2 \pi \sigma^{2} d s$. La force apparente est donc:

$$
\left(2 \pi \sigma^{2}-\frac{1}{8 \pi} \frac{\mathrm{K}-1}{\mathrm{~K}} \mathrm{~F}^{2}\right) d s
$$

Comme on a, près du conducteur, $\mathrm{F}=4 \pi \sigma$, l'expression précédente s'écrit:

$$
\frac{2 \pi \sigma^{2}}{\mathrm{~K}} d s
$$

ce qui est bien le résultat exact.

Ainsi, nous rendons compte, non seulement des attractions et répulsions apparentes des conducteurs électrisés, mais aussi des forces apparentes dues à leur électrisation, et tendant à faire varier leur forme ou leur volume, qui sont, à charges données, en raison inverse de $\mathrm{K}$, comme les premières.

En résumé, la théorie classique est d'accord avec l'expérience. La force électrostatique est partont la même; il s'y joint, dans les diélectiques fluides, une pression hydrostatique qui est un effet indirect, et intervient pour sa part dans les furces apparentes. 